\title{
Assessment of manufacturing process efficiency in the dispersion of carbon fibers in smart concrete by measuring AC impedance
}

Hamza Allam', François Duplan', Sofiane Amziane ${ }^{2}$, Yves Burtschell ${ }^{1}$

${ }^{1}$ Aix-Marseille Université, CNRS, IUSTI UMR 7343, 13453, Marseille, France

2 Univ. Clermont Auvergne, CNRS, Sigma, Institut Pascal, UMR 6602, Clermont-Ferrand, France

\section{HIGHLIGHTS}

- AC impedance measurement is an efficient tool to assess fiber dispersion in mortar

- The protocol of component addition is proved to influence the final impedance and homogeneity of mortar

- When the mixing energy is doubled, impedance values are increased, having a negative effect on the fibers' efficiency in creating conductive paths

- When the percolation increases from around the theoretical percolation threshold to a saturated regime, the effect of the manufacturing process was reduced

\begin{abstract}
Smart concrete is a construction material designed to perform structural and self-sensing roles simultaneously. Self-sensing behavior stems from the inclusion of conductive fibers within the cementitious matrix, reducing its electrical impedance and enabling piezoresistive behavior. Good dispersion of fibers is essential to ensure good mechanical and sensing properties. This study assesses the efficiency of different mixing sequences and energies in dispersing fibers within the mortar. The efficiency is assessed with $0.1 \%$ and $0.5 \%$ carbon fiber volume, by measuring the AC impedance: those volume fractions correspond to two fiber percolation states: around the percolation threshold $(0.1 \%)$, and in the saturation zone $(0.5 \%)$. The study showed that the mixing sequence has a significant effect on the final impedance of the material, and that increasing mixing energy is relatively ineffective in enhancing the fiber dispersion. Differences between different mixing sequences were evident with $0.1 \%$ carbon fibers, while, to
\end{abstract}


a certain extent, saturating the system with conductive fibers reduced the gaps between different mixing sequences.

Keywords: smart concrete, manufacturing process, carbon fibers, electrical impedance, fiber dispersion

\section{Introduction}

Fibrous cement-based materials present beneficial properties compared to an ordinary cementitious matrix. In mechanical terms, the presence of fibers enhances the ductility of the composites [1][2], tensile and flexural strength [3]. In electrical terms, the presence of conductive fibers in the cementitious materials contributes to piezoresistive behavior, which can be used to detect external mechanical loads [4].

The efficiency of carbon fibers cannot be guaranteed if they are not well dispersed in the matrix since, contrary to what is intended, poor dispersion causes negative secondary effects, which deteriorate the matrix instead of enhancing it. The presence of fibers has been proved to affect rheology - especially when clusters are formed. Also, the creation of clusters causes additional air void content, and consequently reduces the compressive strength [5][6]. For sensing behavior, non-homogeneous dispersion of fibers reduces the efficiency of fibers as doping agents and, more importantly, leads to unstable electrical behavior. This instability of initial resistivity values accentuates uncertainties in the sensing behavior of the fibrous mortar.

The mixing protocol appears to play a crucial role in significantly enhancing the dispersion of fibers: the shear velocity is used to spread minor components throughout the matrix to obtain good spatial distribution. In addition, it helps to dissolve agglomerations of fibers, liberating them to create conductive paths more efficiently. On this basis, it appears crucial to adopt a suitable mixing protocol that ensures efficiency in dispersing and distributing the fibers.

Many methods to assess the efficiency of mixing sequences in dispersing fibers have been developed in previous studies. Those methods focus on evaluating fiber dispersion, fiber breakage, fiber segregation and orientation. To assess the state and dispersion of fibers, such methods could be visual, providing a clear, real time image of fibers dispersion [7] [8][9][10][11]. This technique requires powerful scanning tools to 
ensure reliable photo resolution and cover the representative elementary volume (REV). Although, macroscale responses could be effective in evaluating dispersion, as in the "fresh mixture method" [7] applied to fresh cementitious materials, providing indicators as to the global dispersion of the fibers and even their breakage [12]. Other methods, such as the electrical resistance method, provide a macroscale image of the state of the fibers. Electrical resistance is supposed to be a good dispersion criterion, as carbon fibers are more conductive than cement paste, so reduced resistivity would indicate that percolated pathways have been created inside the matrix.

Wang [11] used an X-ray computed tomography (CT) scanner to evaluate the dispersion of carbon fibers in cement-based composites. The grayscale of the pixels was used to differentiate zones as bundles of fibers, uniformly dispersed fibers, or a non-fibrous zone. Wang concluded that in the presence of $2 \%(0.16 \%$ of total volume) of carbon fibers (in relation to the cement weight), fillers were uniformly dispersed through $84-94 \%$ of the mortar's volume, according to this method. The fiber dispersion depends on the materials used (the fiber volume fraction, the presence of dispersion agents, superplasticizers, matrix formulation) and mixing sequence (mixing energy, the order in which the components are added). Wang et al. [8] studied the carbon fiber dispersion $(0.5 \%$ of cement weight) using four different verification methods. With the fresh mixture method, relative standard deviation reaches its lowest value at 2 minutes of mixing. It was assumed that less than 2 minutes of mixing is not sufficient to disperse fibers, while mixing for more than 2 minutes pulls fibers closer together to form clusters and increases fiber breakage. Stroeven [9] used imaging analysis on fibrous mortar slices to study the dispersion of fibers. He showed significant interaction between the fiber content, vibration time and superplasticizer content. Based on the images of cement-based composites, fiber projections were spotted, and their presence is shown either by simple reproduction or by showing the fibers' intersection points. It was noted that increasing the volume fraction of carbon fibers increases boundary effects by providing a higher concentration in the middle of the sample. Excessive vibrating does not seem to enhance the fiber dispersion, and increasing superplasticizer reduces the segregation effect by concentrating the fibers at the core of the specimens. Aldahawi [13] used AC impedance measurement at $1 \mathrm{kHz}$ to globally assess fiber dispersion. Lower resistivity was found to be 
a key criterion for better fiber dispersion. Two methods were tested for the dispersion of carbon fibers (length 6 and $12 \mathrm{~mm}$, fiber volume fraction $=0.5 \%$ ). By mixing the fibers with dry materials before adding water and dispersing agent, lower resistivity values - i.e., better dispersion - was achieved.

Considering the importance of fiber dispersion on their efficiency as doping agents, and consequently in developing piezoresistive behavior, this study uses AC impedance measurement between $4 \mathrm{~Hz}$ and $1 \mathrm{MHz}$ to assess fiber dispersion in mortar in two fiber percolation states: a low-fiber mortar with a $0.1 \%$ carbon fiber volume fraction and a saturated fiber mortar with a $0.5 \%$ carbon fiber volume fraction. The fibers' interaction with sand particles, the liquid phase and the shear velocity of the mixer will determine the final fiber dispersion, distribution, and fiber rupture. In each case, the tested mixing sequences prioritize one or several of the interactions to disperse fibers. Two parameters involved in the mixing sequence are tested: the order of component addition and the mixing energy. Four potential mixing sequences, adopted in previous studies, are tested. Two mixing energies were tested for every mixing sequence. The results will facilitate better understanding of the interaction between mixing and the status of the fibers (dispersion, distribution and rupture) within the mortar. The key parameters are the homogeneity of fibrous mortar, reproducibility of mixes, and efficiency of fibers in creating conductive pathways. A mixing procedure ensuring those parameters would be considered efficient. The measured impedance will be addressed using two approaches: a statistical approach considering mainly the standard deviation between specimens and the reproducibility factor between mixes, and a physical approach assessing the Nyquist plot of fibrous mortar to determine the efficiency of carbon fibers in driving the transition from an insulative cementitious matrix to a conductive composite as a result of mixing procedure.

\section{Methods and experimental setup}

\subsection{Materials}

The carbon fibers used are produced by Toho Tenax; they are $6 \mathrm{~mm}$ in length and $7 \mu \mathrm{m}$ in diameter. Their surface has not been treated with any polymer impregnation. The cementitious matrix is made with CALCIA CEMI $52.5 \mathrm{R}$ cement, standard sand conforming to EN 196-1, respecting the particle size 
distribution (PSD) presented in Table 1, and polycarboxylate-based superplasticizer BASF MasterGlenium ACE 550, the volume of which is increased proportionally to fiber volume fraction to ensure acceptable workability, not exceeding $4 \%$ of cement weight to avoid bleeding. The same $\mathrm{W} / \mathrm{C}$ ratio $(0.4)$ and the same sand volume fraction $(40 \%)$ - i.e., a sand-to-cement ratio of 1.27 - are adopted for all mixes. The mix proportions are summarized in Table 2.

Table 1: Sand particle size distribution

\begin{tabular}{|c|c|}
\hline Diameter $(\mathrm{mm})$ & Cumulative particle passing percentage $(\%)$ \\
\hline 0.063 & 0.03 \\
\hline 0.125 & 2.41 \\
\hline 0.25 & 24.86 \\
\hline 0.5 & 34.19 \\
\hline 1 & 68.81 \\
\hline 1.6 & 100 \\
\hline
\end{tabular}

Table 2: Mix proportions of the mortar

\begin{tabular}{|c|c|c|c|c|c|}
\hline & Cement & Sand-to- & Water-to- & Superplasticizer & Fiber volume \\
& content $(\mathrm{g} / \mathrm{l})$ & cement ratio & cement ratio & ratio-to-cement & fraction (\%) \\
& & & & weight $(\%)$ & \\
\hline Low-fiber & 830 & 1.27 & 0.4 & 0.72 & 0.1 \\
\hline $\begin{array}{c}\text { Hortar } \\
\text { High-fiber }\end{array}$ & 830 & 1.27 & 0.4 & 1.81 & \\
\hline
\end{tabular}

The next section explains the mixing of the components. The mixing sequence and energy were tested to evaluate their effect on fiber dispersion. To assess the relationship between the impact of mixing on 
impedance and the theoretical percolation state of carbon fibers, each mixing sequence was tested for a low-fiber volume fraction - $0.1 \%$ (around the percolation threshold of carbon fibers) and a high-fiber volume fraction - $0.5 \%$ (in the saturation zone of carbon fibers) [6].

Fibrous mortar was then poured into molds, creating three samples measuring $4 \times 4 \times 16 \mathrm{~cm}$. The samples were unmolded at 24 hours, then immersed at $20^{\circ} \mathrm{C}$ for 28 days of wet curing. Next, they were placed in a controlled environment at $20{ }^{\circ} \mathrm{C}$ and $50 \% \mathrm{RH}$ for 28 days, and finally, their electrical impedance was measured.

\subsection{Experimental setup}

The AC impedance of samples was measured between $4 \mathrm{~Hz}$ and $1 \mathrm{MHz}$; within this range, the measurements were recorded at 33 frequency points per decade, (totaling 200 frequencies over the entire range), using a Hioki IM3750 impedance analyzer with a voltage of $0.1 \mathrm{~V}$. The electrodes were copper plates, affixed to the $4 \times 4 \times 16 \mathrm{~cm}$ samples with conductive adhesive copper foil installed on conductive nickel paint applied to the ends of the dried samples after careful polishing [14]. A two-electrode configuration was adopted. Measurements were performed after 28 days of wet curing and a further 28 days of dry curing at $20{ }^{\circ} \mathrm{C}$ and $50 \% \mathrm{RH}$ in a controlled environment.
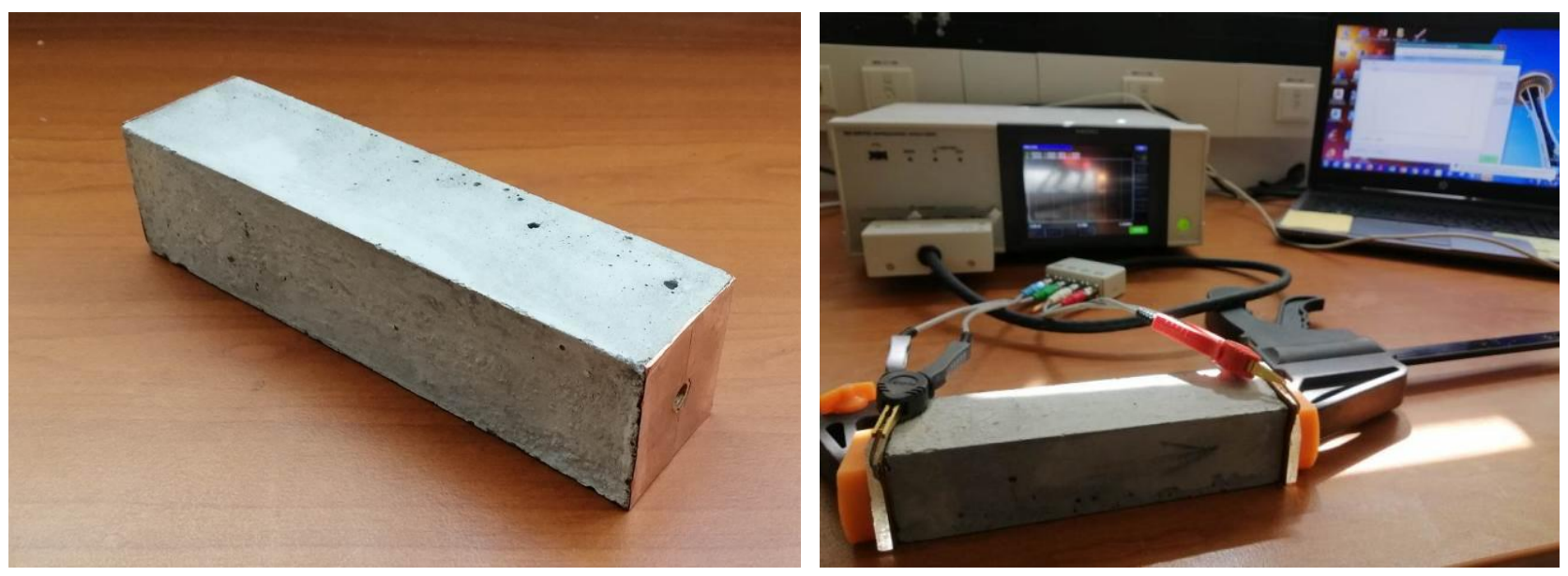

Fig. 1: Measurement setup

\subsection{Mixing protocol}

Four mixing sequences were tested, as detailed in Table 3. They were labelled "One step", "Dry mix", "Wet mix 1" and "Wet mix 2", depending on where in the sequence the fibers were added. 
Every scenario presents specific advantages and drawbacks in terms of the dispersion of fibers. To avoid variation of the mixing energy, all mixing sequences were tested, totaling 3 minutes of mixing across at a constant velocity $\mathrm{v}=140 \mathrm{rpm}$ (low energy, low velocity - LELV).

Table 3: Mixing sequences

\begin{tabular}{|c|c|c|c|c|}
\hline Time axis (s) & Onestep.LELV & Drymix.LELV & Wetmix1.LELV & Wetmix2.LELV \\
\hline 0 & All components & $\begin{array}{l}\text { Dry components } \\
\text { (C+S+CF) }\end{array}$ & $\mathrm{C}+\mathrm{S}$ & $\mathrm{C}+\mathrm{S}$ \\
\hline \multicolumn{5}{|c|}{$1 \mathrm{~min}(140 \mathrm{rpm})$} \\
\hline 60 & & $\begin{array}{l}\text { Wet components } \\
\qquad(\mathrm{W}+\mathrm{SP})\end{array}$ & $\begin{array}{l}\text { Wet components } \\
\qquad(\mathrm{W}+\mathrm{SP})\end{array}$ & $\mathrm{W}+\mathrm{SP}+\mathrm{CF}$ \\
\hline \multicolumn{5}{|c|}{$1 \mathrm{~min}(140 \mathrm{rpm})$} \\
\hline 120 & & & $\mathrm{CF}$ & \\
\hline \multicolumn{5}{|c|}{$1 \mathrm{~min}(140 \mathrm{rpm})$} \\
\hline
\end{tabular}

\subsection{Mixing energy}

As per previous studies [15], the mixing energy $E$ is calculated by:

$$
\mathrm{E}=\mathrm{C}^{*} \Omega^{*} \mathrm{t}
$$

where $C$ is the torque applied by the flat beater (N.m), $\Omega$ is the rotational velocity of the mixer and $t$ is the mixing time. The torque $\mathrm{C}$ is proportional to the rotational velocity $\Omega$ :

$$
C=K^{*} \Omega^{*} \rho
$$

where $\mathrm{K}$ is an experimental value $\left(=6.4 * 10^{-9} \mathrm{~N} \mathrm{~m} / \mathrm{kgm}^{3} / \mathrm{rpm}\right)$ in the case of cementitious materials, and $\rho$ is the specific weight. The specific mixing energy (SME) is then calculated as follows:

$$
\mathrm{SME}=\frac{E}{m}=\frac{k * \Omega^{2} * t}{V}
$$

where $\mathrm{V}$ is the corresponding volume. 
For the low mixing energy, $\Omega=140 \mathrm{rpm}$, and $\mathrm{t}=3$ minutes (LELV). Mixing energy is doubled to assess its variation effect on impedance. This doubling of mixing energy is accomplished either by increasing the final mixing velocity (high energy high velocity - HEHV) or extending the final mixing time at low mixing velocity (high energy low velocity - HELV). Different mixing energies were applied to the four mixing protocols listed above. The case of different mixing energies of dry mix is shown in Table 4 as an example.

Table 4: Mixing energy protocol

\begin{tabular}{|c|c|c|c|}
\hline Time axis (s) & Drymix.LELV & Drymix.HELV & Drymix.HEHV \\
\hline 0 & $\begin{array}{l}\text { Dry components } \\
\qquad(\mathrm{C}+\mathrm{S}+\mathrm{CF})\end{array}$ & $\begin{array}{l}\text { Dry components } \\
\qquad(\mathrm{C}+\mathrm{S}+\mathrm{CF})\end{array}$ & $\begin{array}{l}\text { Dry components } \\
\qquad(\mathrm{C}+\mathrm{S}+\mathrm{CF})\end{array}$ \\
\hline \multicolumn{4}{|c|}{$1 \mathrm{~min}(140 \mathrm{rpm})$} \\
\hline 60 & $\begin{array}{l}\text { Wet components } \\
\qquad(\mathrm{W}+\mathrm{SP})\end{array}$ & $\begin{array}{l}\text { Wet components } \\
\qquad(\mathrm{W}+\mathrm{SP})\end{array}$ & $\begin{array}{l}\text { Wet components } \\
\qquad(\mathrm{W}+\mathrm{SP})\end{array}$ \\
\hline \multicolumn{4}{|c|}{$1 \mathrm{~min}(140 \mathrm{rpm})$} \\
\hline \multirow[t]{2}{*}{120} & & & \\
\hline & $1 \mathrm{~min}(140 \mathrm{rpm})$ & $4 \mathrm{~min}(140 \mathrm{rpm})$ & $1 \mathrm{~min}(280 \mathrm{rpm})$ \\
\hline
\end{tabular}

\subsection{Methods: Assessment of fiber dispersion}

\subsubsection{Statistical approach}

In assessing the fiber dispersion using electrical measurements, two parameters were adopted as key assessment criteria: the average electrical resistivity $\bar{\rho}$, and standard deviation $\sigma$, calculated as follows:

$$
\begin{gathered}
\bar{\rho}=\frac{1}{n} \sum \rho_{\mathrm{i}} \\
\sigma=\sqrt{\frac{\sum(\rho \mathbf{i}-\bar{\rho}) 2}{n}}
\end{gathered}
$$

where $\mathrm{n}$ is the number of specimens, and $\rho_{\mathrm{i}}$ is the resistivity of the specimen $\mathrm{i}$. 
A low $\bar{\rho}$ value would prove the fibers are efficient in creating conductive pathways, which cannot be guaranteed if those fibers are not well dispersed within the matrix. A low $\sigma$ is an indicator of homogeneity of the mix, reflecting similarity between specimens in terms of measured impedance. Homogeneous material means fiber well dispersed inside the cementitious matrix. Fibers well dispersed in the cementitious matrix means very small differences between different volumes of the same prepared batch. Very small differences would lead to small differences between samples of the same patch, i.e. small standard deviation $(\sigma)$ values are a sign of homogeneity.

Another mixing efficiency factor is added in this study to evaluate mixing reproducibility; the mixes are reproduced twice, and reproducibility is assessed with the factor $\mathrm{k}$ :

$$
k=\left|\rho_{1}-\rho_{2}\right|
$$

where $\rho_{1}$ and $\rho_{2}$ are the respective impedances of the two mixes.

An efficient mixing sequence is, for the same formulation, a reproducible sequence. A reproducible mixing sequence lead to very small differences between repeated batches of the same formulation. Very small

differences between batches lead to the same electrical behavior for repeated batches, i.e. a small $\mathrm{k}$ value is sign of reproducible mixing sequence.

These two values $(\sigma$ and $\mathrm{k}$ ) are actually related to the mixing process, the preparation technology of the operator, the homogeneity of the raw materials and the stability of the mixing equipment. In the study the same preparation technology (mixing in the same mixer, that presents the same size and the same flat beater) is used, the same raw material and the same mixing equipment (PERRIER mixer). The only changing parameter is the mixing process. If all the parameters are fixed, we suppose that standard deviation is kept in the same margin. If one parameter is changed-in this study the mixing process - and differences are noticed in standard deviation values for example, a correlation is possible between the

\section{changing parameter and the measured variation.}

The real and imaginary impedances are compared across the whole measured frequency range, and the average standard deviation and reproducibility factors are found to compare different mixing processes. 


\subsubsection{Physical approach}

In this section, the efficiency of carbon fibers in increasing conductivity is the key parameter, where the factor assessed is the percolation state of carbon fibers. The goal is to determine the efficiency of mixing sequences in dispersing fibers through the matrix. This dispersion is evaluated through the lens of AC impedance. The method of AC impedance spectroscopy in evaluating fiber dispersion has been approved in previous studies [16]. In the absence of fibers, the electrical behavior of cement-based materials has been thoroughly studied in previous publications [17]. It is presented on a Nyquist plot (the imaginary impedance is a function of the real impedance), with the typical diluted semi-arc presented in the figure below, that could be fitted to an equivalent R-C circuit.
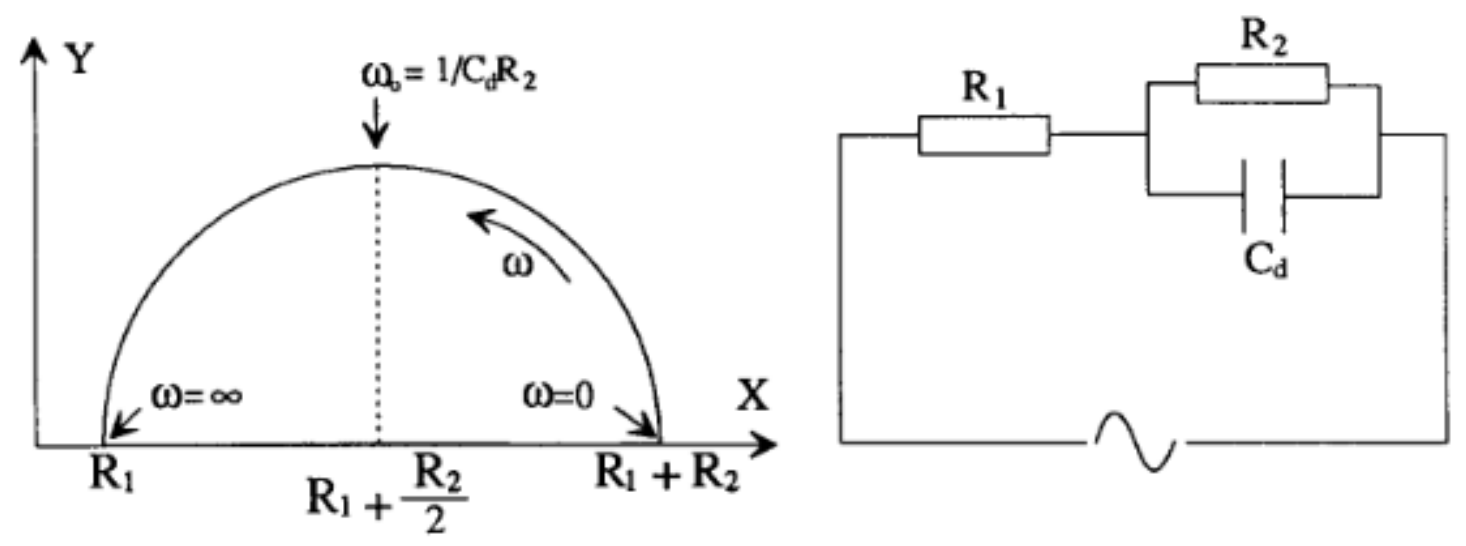

Fig 2: Equivalent R-C circuit and the corresponding Nyquist plot [17]

$$
\begin{aligned}
& Z^{\prime}=R_{1}+\frac{R_{2}}{1+\omega^{2} R_{2}^{2} C^{2}} \\
& Z^{\prime \prime}=-\frac{w R_{2}^{2} C}{1+\omega^{2} R_{2}^{2} C^{2}}
\end{aligned}
$$

where $Z^{\prime}$ is the real impedance, $Z^{\prime \prime}$ is the imaginary impedance, and $\omega=2 \pi f$.

The diameter of the perfect semi-circle could be equal to $R_{2}$ and the peak value $=R_{2} * C$. The relaxation frequency corresponding to the imaginary peak would be $f_{\text {peak, }}$ where $R C=1 / 2 \pi f_{\text {peak }}$.

The presence of carbon fibers in the cementitious matrix would create conductive paths, leading to the transition of mortar from an insulative material to a conductive one. Higher aspect ratio (less fiber rupture during mixing) [18] and better dispersion [19] promote the efficiency of fibers in increasing conductivity. Those percolated paths create parallel pathways, increasing conductivity and thus reducing global 
impedance. This arrangement can be reflected in the equivalent circuit by adding a parallel R-C circuit [20], with smaller $\mathrm{R}$ and $\mathrm{C}$ values, considering the conductive nature of the fibers. Thus, the conductivity paths would affect the Nyquist plot of the composite: reducing the semi-arc diameter and reducing the peak value. A second semi-arc would be created at high frequencies to represent the conductive path response; however, this effect is not taken into consideration in this study. In the ultimate case of fiber percolation, AC impedance would be dominated by the fibers' contribution, exhibiting quasi-resistive behavior. The corresponding equivalent circuit is very close to a simple resistor, with $Z^{\prime}$ related mainly to the real impedance of the percolated pathways and Z" significantly reduced.

In this case, the Nyquist plot is very similar to a single point on the real axis, with the abscissa $=Z^{\prime}$ dominated by conductive paths and $Z^{\prime \prime}$ significantly reduced and approaching zero. The effectiveness of mixing procedure in dispersing carbon fibers and avoiding their rupture can be tested based on the progression, due to the presence of fibers, in the transition from an $\mathrm{R}-\mathrm{C}$ insulative circuit to resistive behavior. The following criteria are adopted to assess the progress:

- Diameter of the Nyquist arc: the distance between the cusp frequencies (frequencies with minimal imaginary values) is calculated. A smaller distance means a smaller gap in real impedance across the frequency range - i.e., more conductive behavior (this criterion is designed especially to compare two impedances in samples at the same age - i.e., which theoretically have the same water content). A smaller arc diameter means the fibers are more effective.

- Imaginary peak values detected at relaxation frequencies: smaller peaks (as explained above) mean that the conductive fillers are efficient in creating parallel conductive paths through the insulative matrix, reducing the measured imaginary impedance.

\section{Results}

The different impedance responses measured after 28 days of wet curing of the samples and a further 28 days of dry curing at $20^{\circ} \mathrm{C}$ and $50 \% \mathrm{RH}$ are presented in this section 3 .

\subsection{Mixing protocol}




\subsubsection{Low-fiber mortar}

Fig. 3 illustrates the real and imaginary impedances of low-fiber mortar as a function of the frequency range. Mixing protocols appear to have a significant effect on impedance values. In all mixing sequences, real impedance decreases as the frequency increases. This finding is consistent with the real impedance expression given above (7). It could be attributed to the reduction in the polarization effect as the frequency increases [21]. The one-step mixing sequence exhibited the highest real impedance values, starting from $4 \times 10^{2} \Omega$ at low frequencies, while the real impedance values for the three other mixing sequences were significantly lower, at around $2 \times 10^{2} \Omega$. The difference persisted with frequency variation, and again, the highest impedance values were found with the one-step method. Regardless of mixing method, we always saw a particular relaxation frequency where the imaginary impedance reached its maximum. Once again, this is in keeping with expression (8) of the imaginary impedance. The values of the imaginary impedance across the frequency range depend on the mixing sequence: The one-step mixing protocol yielded the highest imaginary values throughout the frequency range, especially at the relaxation frequency, where, even in the presence of fibers, imaginary impedance reaches $82 \Omega$ between $100 \mathrm{~Hz}$ and $1 \mathrm{kHz}$. For the other mixing sequences, the imaginary impedance across the whole frequency range is lower, and the peak imaginary impedance does not exceed $40 \Omega$ at relaxation frequencies. It is important to note that the relaxation frequency in the one-step method is lower than in other mixing sequences.

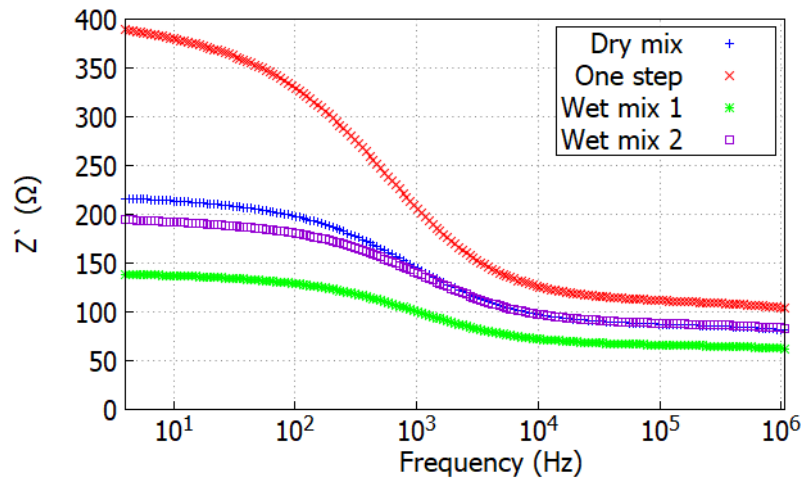

(a)

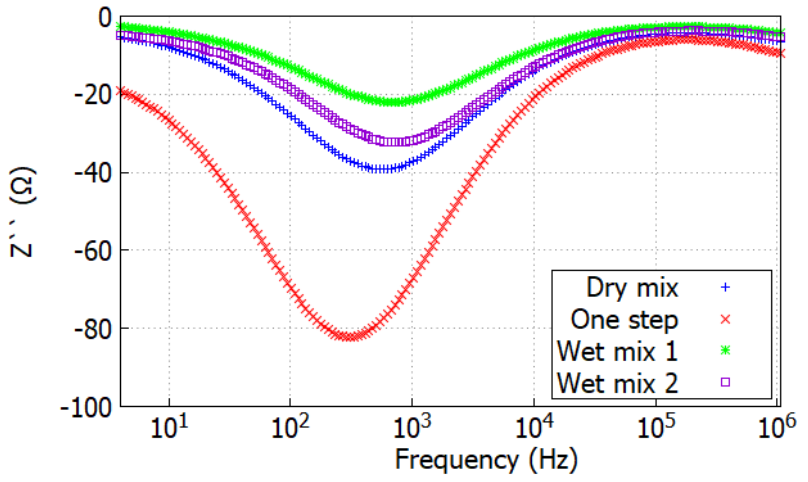

(b)

Fig. 3: Impedance of low-fiber mortar 
The relation between the real and imaginary impedance of fibrous mortar is shown in Fig. 4, on the Nyquist plots for the different mixing protocols. For all mixing sequences, cusp and relaxation frequencies are present in the assessed frequency range. The typical diluted semi-circular arc summarizes the electrical behavior of different fibrous mortars. The measured semi-circles depend upon mixing sequences: the onestep method presents the semi-circle with the biggest diameter, while the other three mixing protocols tend to reduce the diameter of that semi-circle. Similarly, the imaginary impedance peak values are heavily dependent upon mixing sequence: the high peak present with the one-step mixing sequence is significantly reduced with the other mixing protocols.

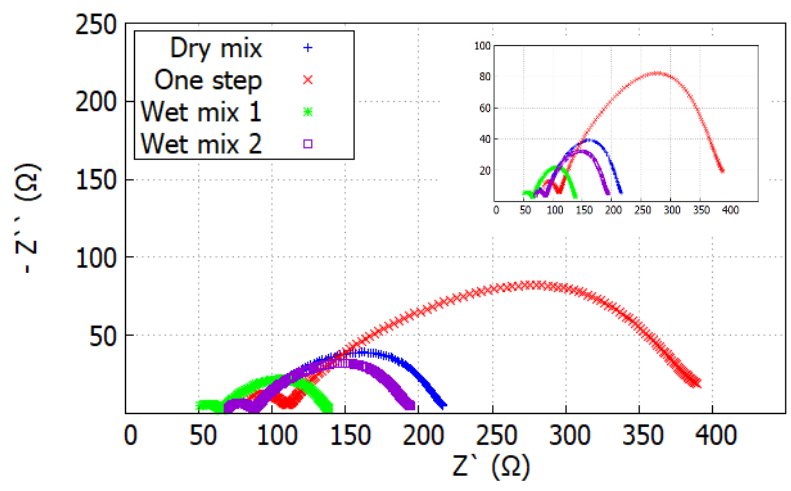

Fig. 4: Nyquist plot of low-fiber mortar

\subsubsection{High-fiber mortar}

Fig. 5 illustrates the impedance of high-fiber mortar with different mixing protocols. Increasing carbon fiber volume fraction, regardless of mixing protocol, has a significant impact on the impedance. It is reduced by many orders of magnitude, emerging in the percolated state. This percolated state is confirmed by previous theoretical [22] and experimental [23] studies. Variation of real impedance over the frequency range is very limited due to the presence of carbon fibers. The behavior is closer to a quasi-resistive behavior. The real impedance values in Fig. 5a are affected, to a considerably lesser extent than with lowfiber mortar, by mixing protocol, not exceeding $2 \times 10^{1} \Omega$, with slightly higher values measured with the one-step and wet mix 2 methods. Peaks in imaginary impedance can still be identified, to a lesser extent than with low-fiber mortar. For different mixing sequences, the absolute values of imaginary impedance, similarly to the real impedance values, are significantly reduced by the presence of fibers. The highest peak 
values are also detected with the one-step and wet mix 2 methods, not exceeding $1.5 \Omega$, slightly higher than the imaginary peak values recorded: around $0.5 \Omega$ with dry mix and wet mix 1 methods.

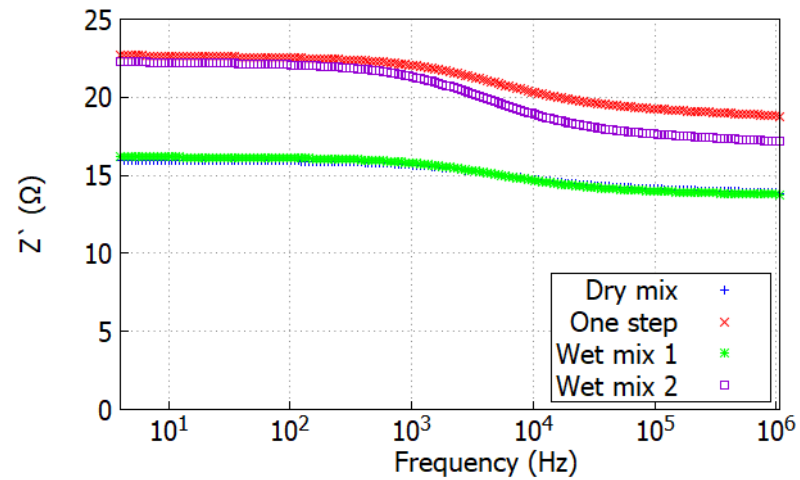

(a)

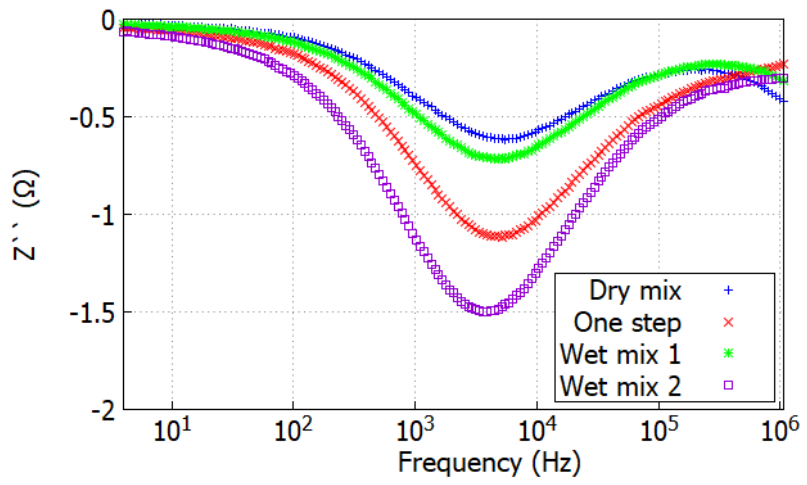

(b)

Fig. 5: Impedance of high-fiber mortar

The Nyquist plots of different mixing sequences are shown in the presence of a high fiber volume fraction. The relation between real and imaginary impedance is summarized in Fig. $\mathbf{6}$. The semi-arc size, in the case of high-fiber mortar, is greatly reduced compared to low-fiber mortar: as stated previously, the behavior of fibrous mortar is closer to a quasi-resistive behavior. For the One-step and Wet mix 2 methods, slightly bigger semi-arcs are detected compared to Dry mix and Wet mix 1.

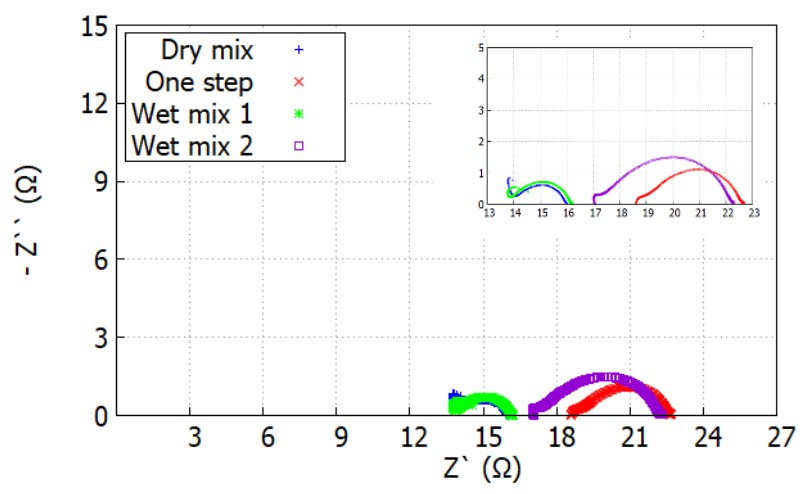

Fig. 6: Nyquist plot of high-fiber mortar

\subsection{Mixing energy}

\subsubsection{Low-fiber mortar}

The mixing energy is increased, as previously mentioned, either by increasing the mixing time, or mixing velocity after the addition of fibers. For different mixing sequences, the effect of increasing the mixing 
energy on the real impedance is presented in Fig. 7. Mixing energy seems to affect real impedance values. Increasing either the mixing time or mixing velocity causes an increase in resistance, and in the best-case scenario, do not seem to reduce it. For different mixing sequences, an increase in real impedance was observed when increasing the mixing energy, but more significant with the One-step method. Furthermore, extending the mixing time at low velocity or increasing the final mixing velocity did not seem to have the same effect. Note that in the case of increased mixing velocity, real impedance increased to a lesser extent, regardless of the mixing sequence.

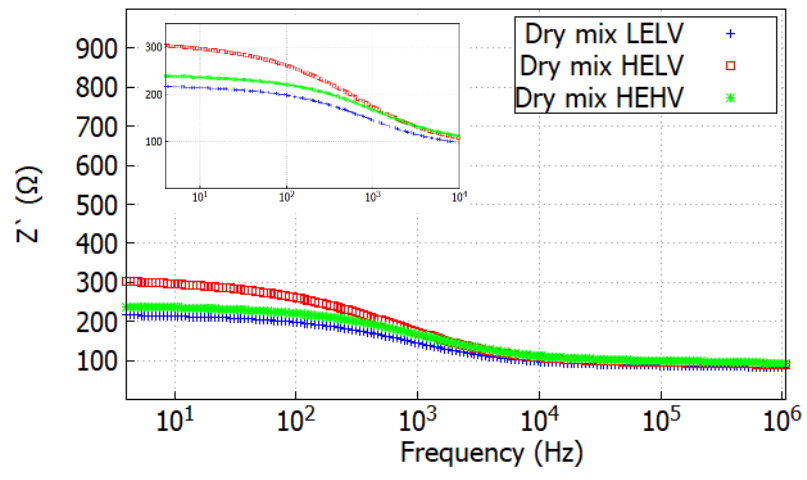

(a)

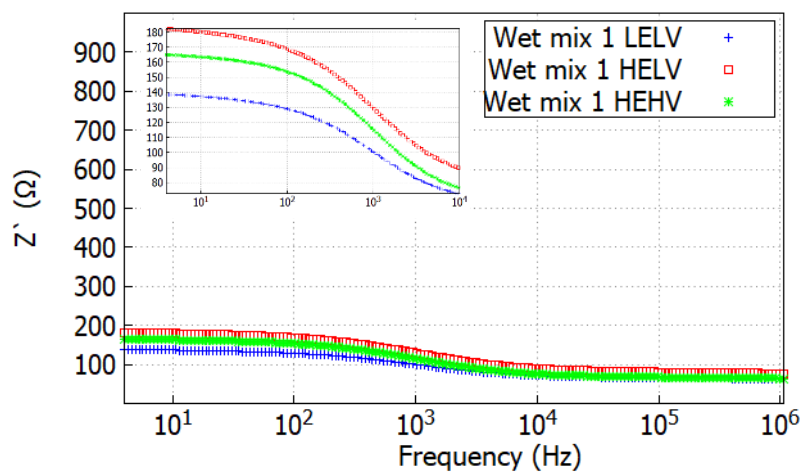

(c)

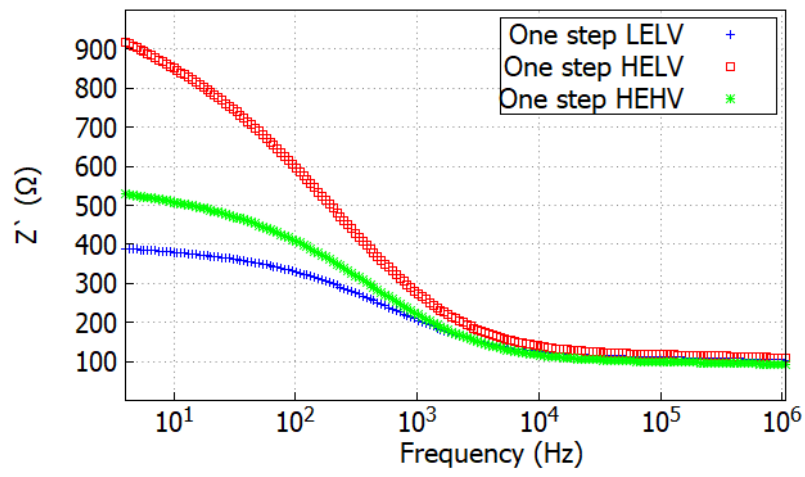

(b)

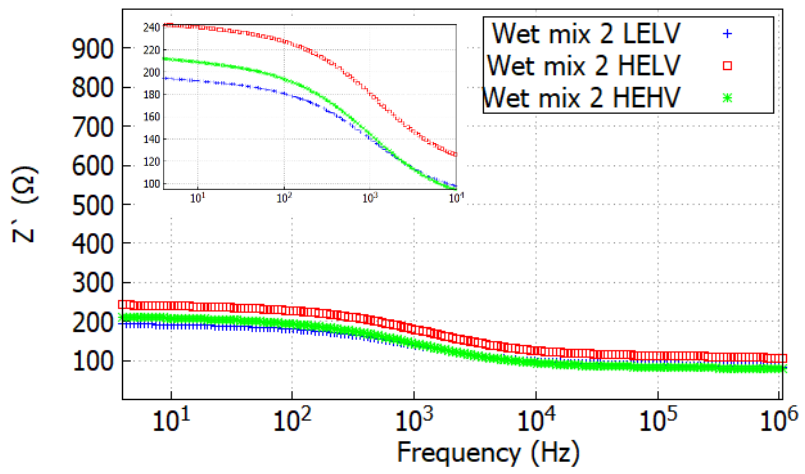

(d)

Fig. 7: Real impedance of low-fiber mortar for different mixing energies

The imaginary impedance, shown in Fig. 8, also demonstrates the effect of mixing energy. With longer mixing time, imaginary impedances are higher across the full frequency range. Peak values at relaxation frequencies are relatively higher than those corresponding to low mixing energy, reached at lower relaxation frequencies. When the final mixing velocity was increased instead, we still found higher 
impedance values than low-energy mortars, but with the One-step and Dry mix methods, it was considerably more effective in reducing the imaginary impedance.

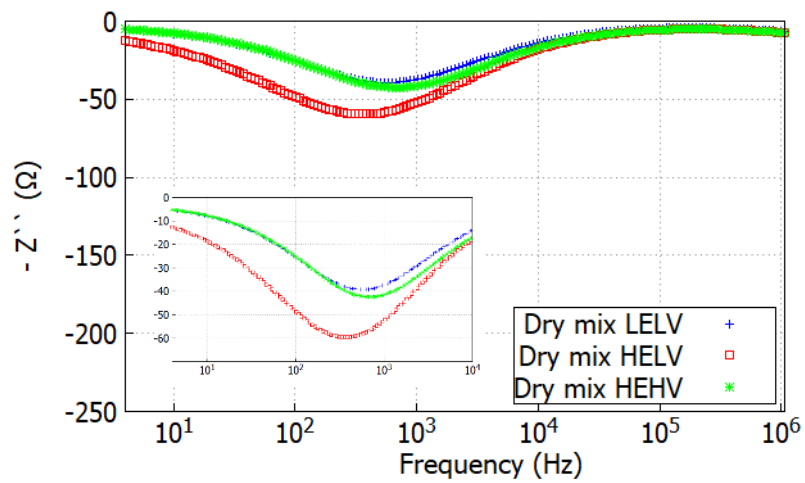

(a)

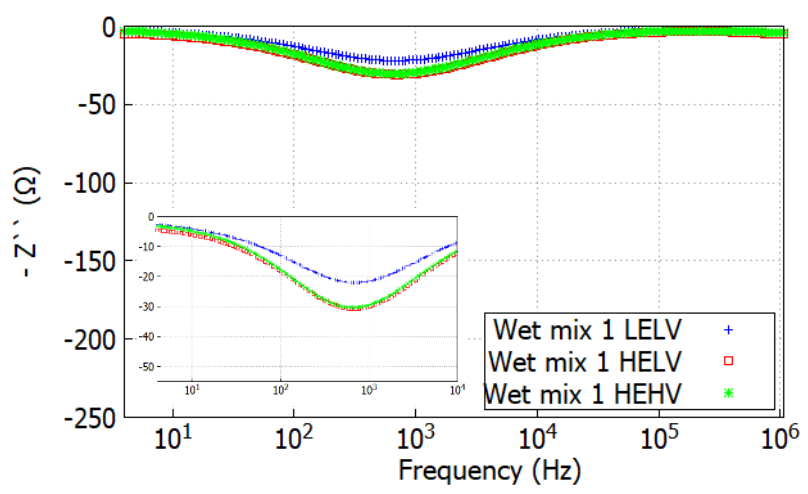

(c)

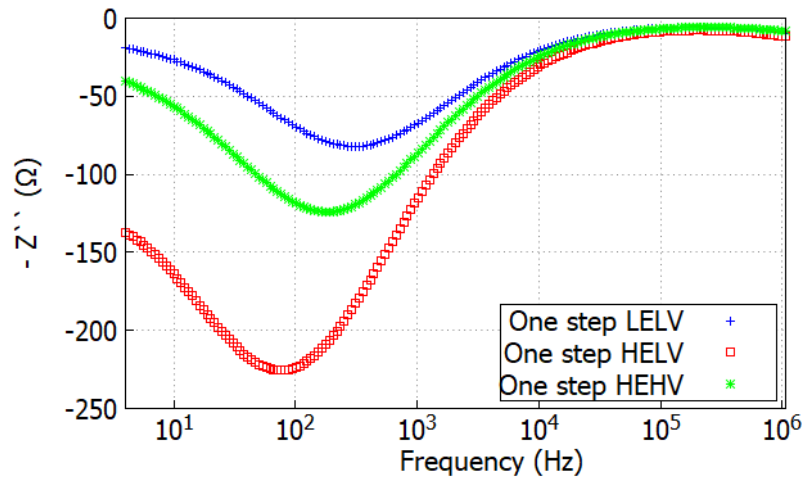

(b)

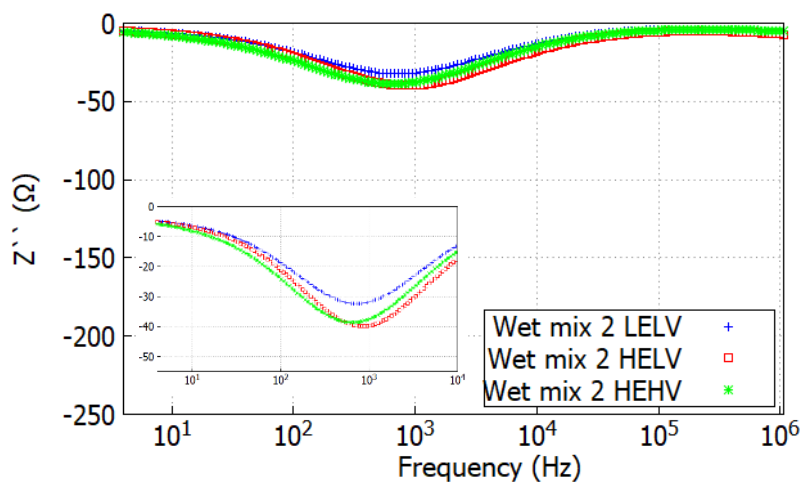

(d)

Fig. 8: Imaginary impedance of low-fiber mortar for different mixing energies

The Nyquist plots presented in Fig. 9 illustrate the relation between the real and imaginary impedance of mortars with different mixing energies, for different mixing protocols. The effects of increasing mixing energy are clear in increasing impedance for most of the mixing protocols. Our earlier observations are reflected on the Nyquist plot of the diluted semi-arcs. The diameter of the semi-arc corresponding to high mixing energy is significantly higher for all mixing sequences. A considerable difference is to be noted between the two protocols of increasing mixing energy: increasing the final mixing velocity instead of extending the mixing time at low velocity seems to be more effective in reducing the semi-arc volume for different mixing protocols. 


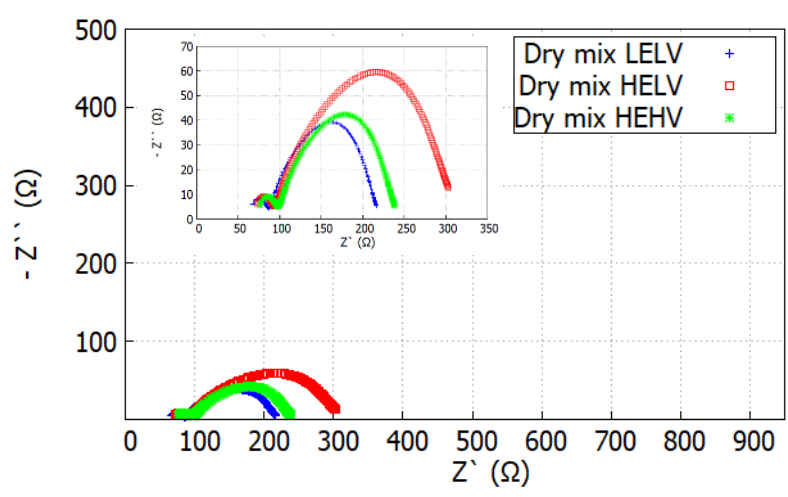

(a)

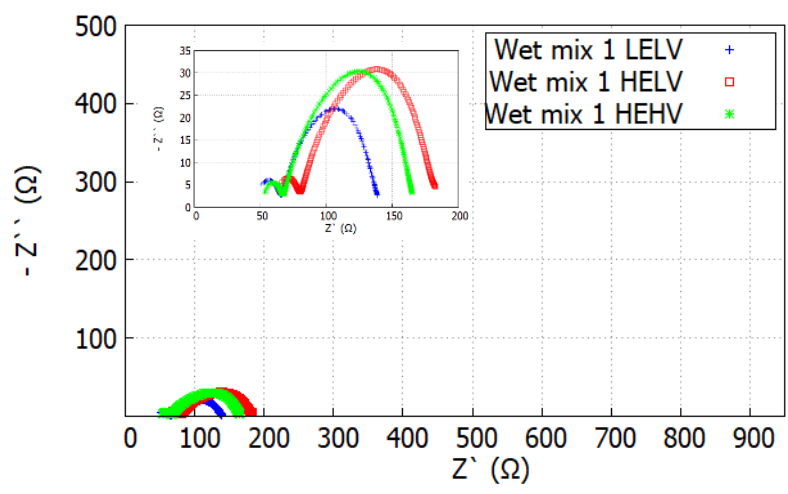

(c)

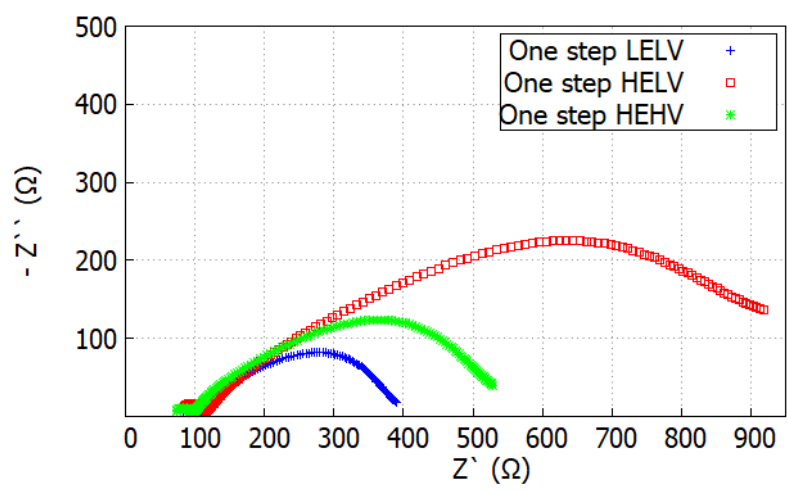

(b)

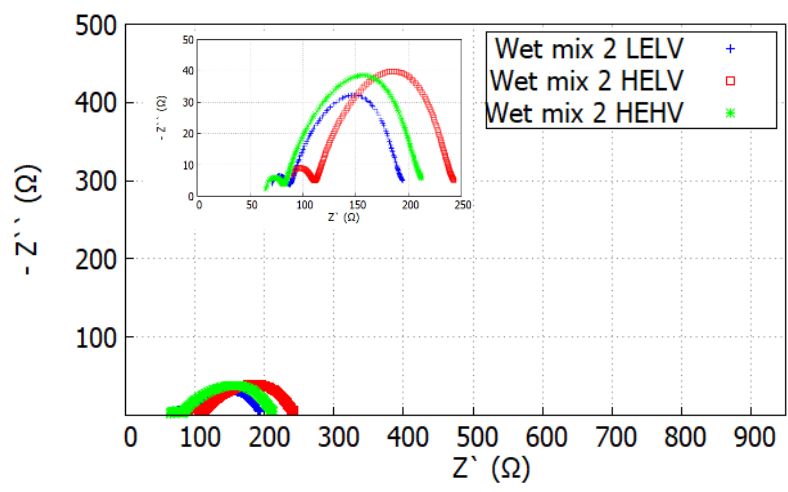

(d)

Fig. 9: Nyquist plot of low-fiber mortar for different mixing energies

\subsubsection{High-fiber mortar}

Mixing energy has a reduced, though still discernible, effect on the resistivity of high-fiber mortar. The effects of mixing energy were moderated by the high fiber content. For real impedance, illustrated in Fig. 10, in the case of low mixing energy, the values for all mixing protocols were quasi-constant throughout the frequency range. Extending the mixing time, especially in the case of the Dry mix and One-step method, increased real impedance values to around $4 \times 10^{2} \Omega$ at low frequencies, and their values became more frequency dependent, but still with a very slight gap between low and high frequencies. Imaginary impedance, illustrated in Fig. 11, was also affected by the variation in mixing energy. When extending mixing time, imaginary values and especially imaginary peaks at relaxation frequency showed a slight increase; this effect was reduced when the mixing velocity, rather than the mixing time, was increased. Differences between mixing energies did not remain for all mixing sequences: for the Wet mix 1 and Wet mix 2 methods, changing mixing energy did not seem to have a significant effect. 


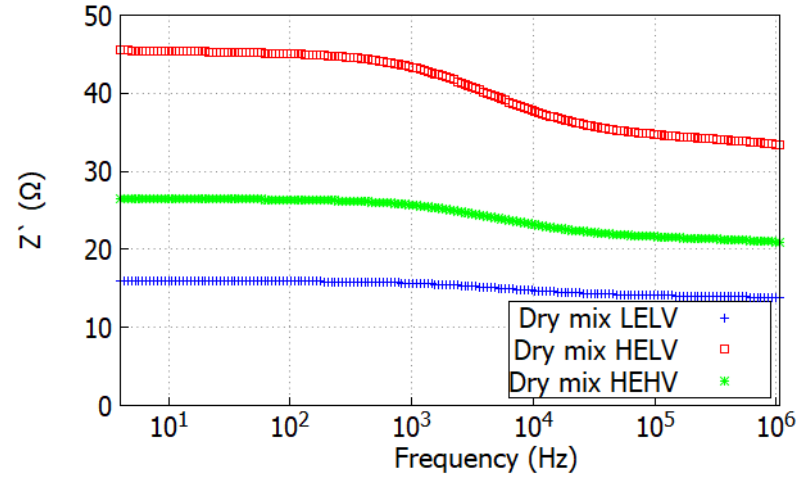

(a)

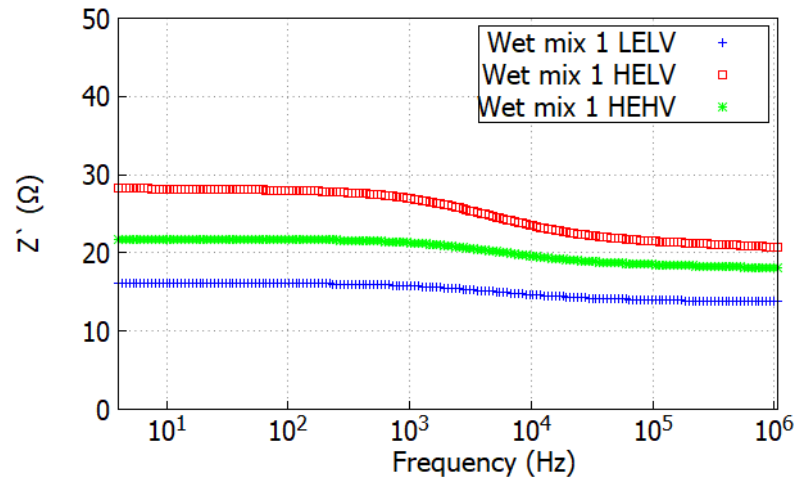

(c)

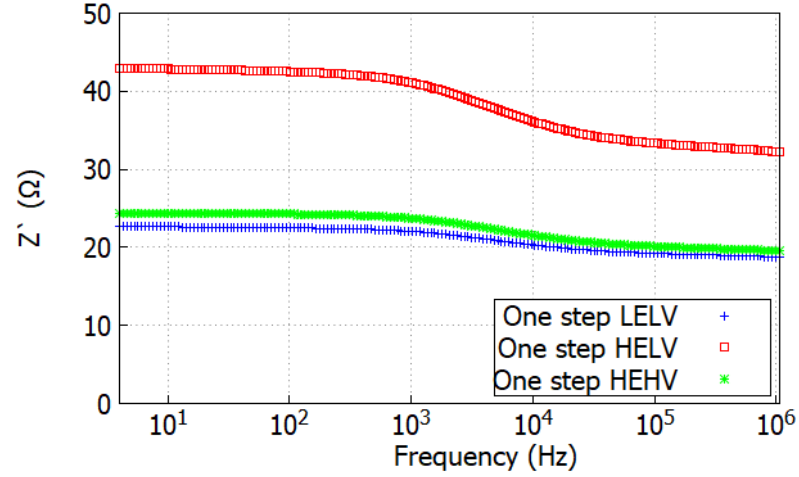

(b)

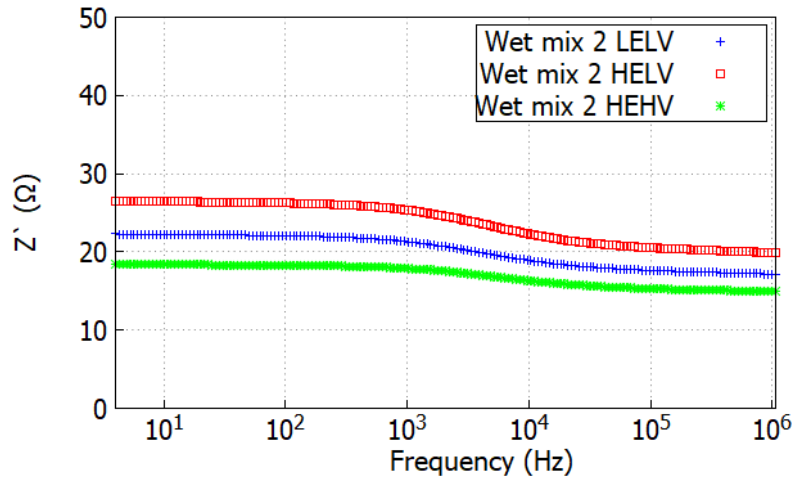

(d)

Fig. 10: Real impedance of high-fiber mortar for different mixing energies

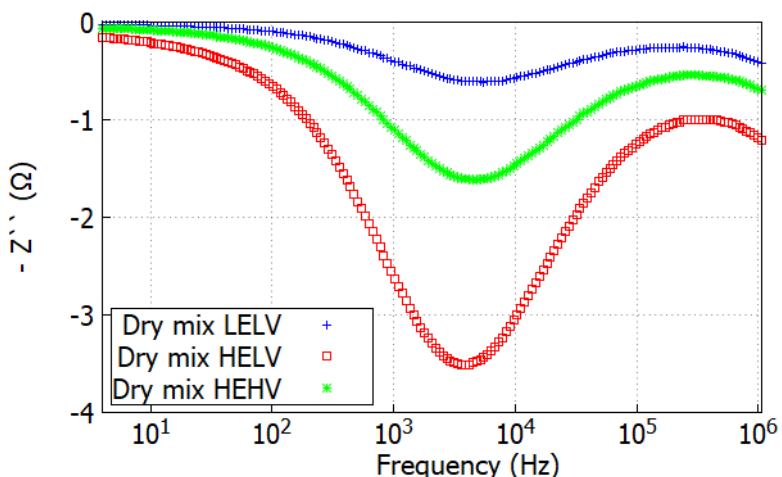

(a)

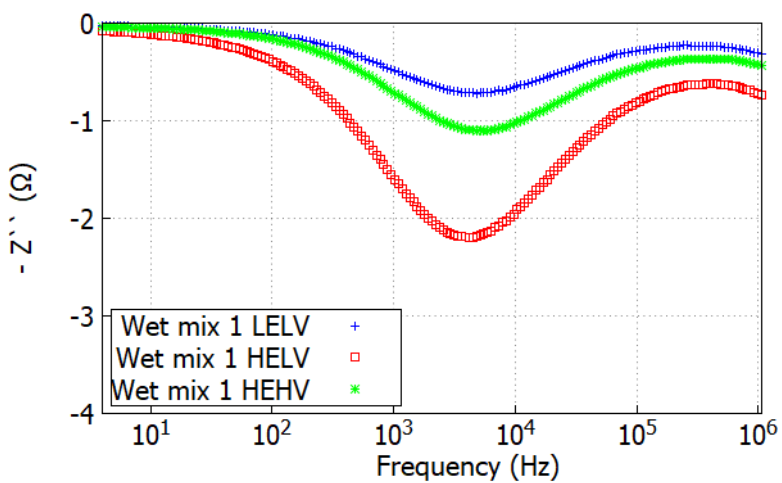

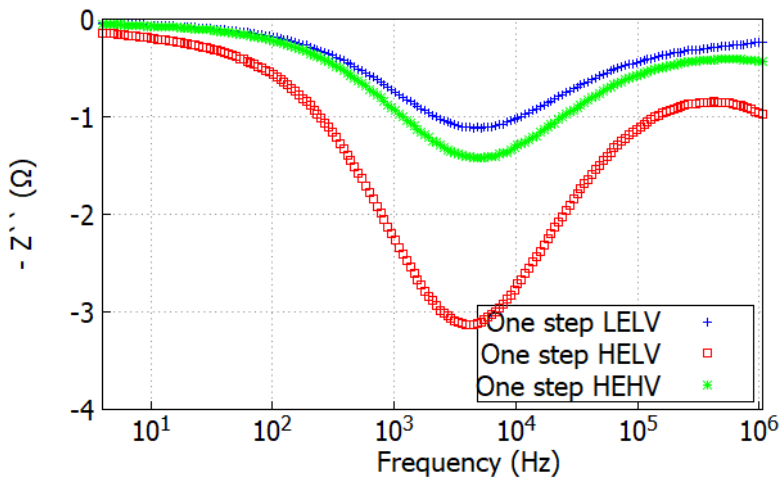

(b)

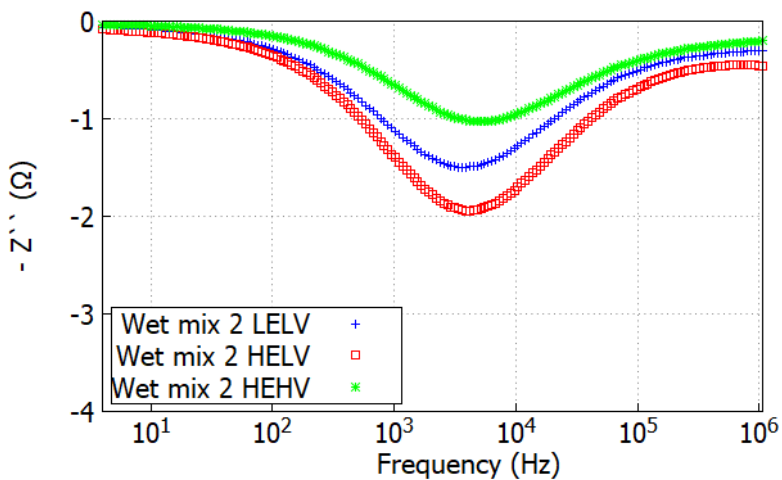


Fig. 11: Imaginary impedance of high-fiber mortar for different mixing energies

The Nyquist plots in Fig. 12 summarize the real and imaginary impedance relation for different mixing energies in high-fiber mortar. The diluted semi-arcs, due to the presence of fibers, are relatively small in diameter, and peak values for different mixing protocols. The differences seem to persist but are less pronounced than in the case of low-fiber mortar.

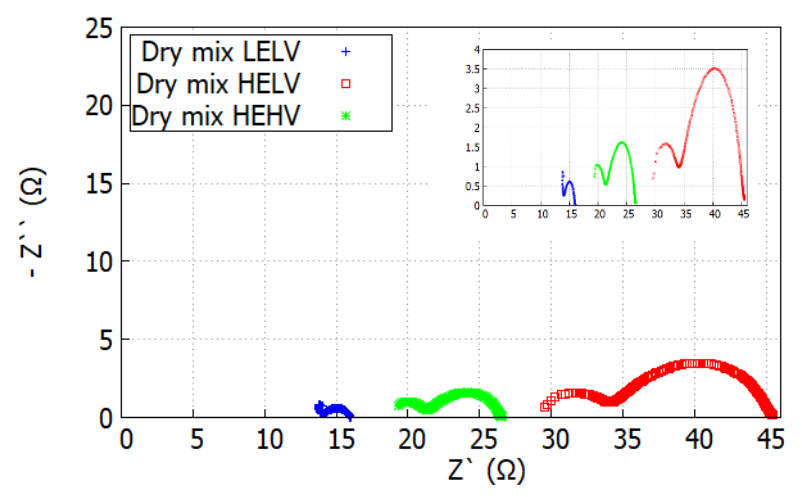

(a)

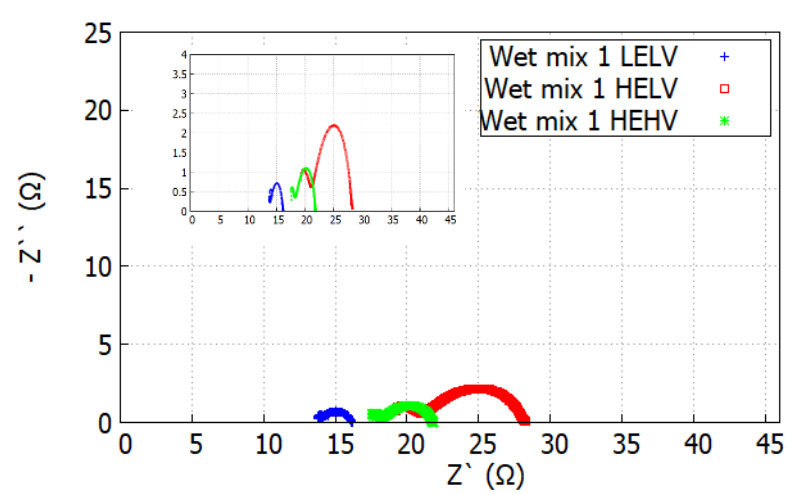

(c)

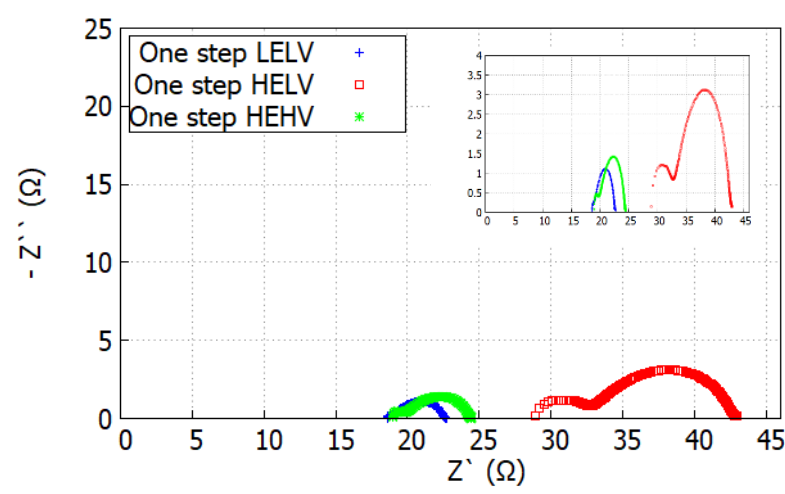

(b)

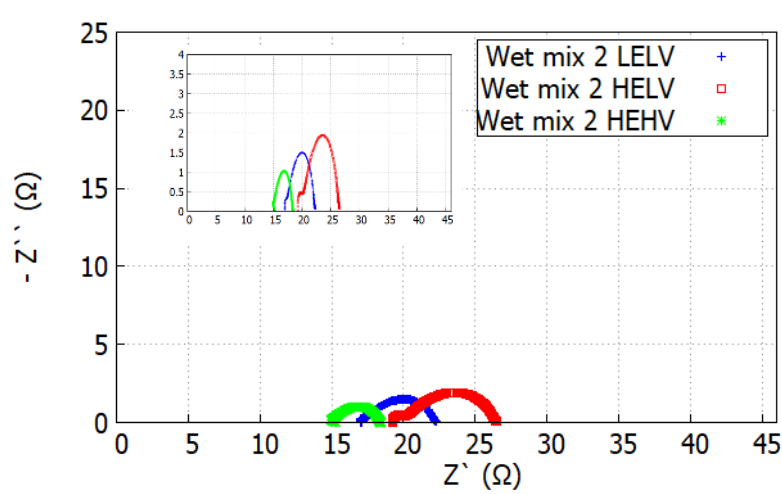

(d)

Fig. 12: Nyquist plot of high-fiber mortar for different mixing energies

\section{Discussion}

As explained previously, this study relates mixing efficiency in dispersing carbon fibers to the electrical impedance of fibrous mortar. Since mixing protocol may play a crucial role in dispersing conductive fibers, it may also dictate electrical response. Mixing protocol influences the distribution of fibers within the 
matrix, fiber clusters and fiber rupture. As regards fiber distribution, a good mixing sequence would ensure better distribution of carbon fibers in the volume of composites, resulting in a better chance of creating conductive paths throughout the volume of the mortar [16], and thus decreasing the volume impedance. Moreover, a good distribution reduces differences between different specimens [7], giving a more homogeneous impedance for different specimens, reflected in a decrease of standard deviation values. The effect of fibers presence on the impedance values, compared to non-fibrous cementitious matrix, would reduce it by many orders of magnitude as proved previously [6].

For fibers length, it was previously stated that mixing sequence causes fiber rupture [12][24]. This fiber length reduction would significantly increase impedance values, due to the reduction of the aspect ratio of fibers, which, according to percolation theory [22] and previous experimental results [25], is a crucial factor in treating percolation. The theoretical estimations of percolation threshold assume random fiber orientation, distribution, and nominal aspect ratio of fibers. Experimental results may not perfectly fulfill all those requirements. Fiber rupture would have a negative effect on the efficiency of the fibrous mortar, increasing the quantity of fibers needed to reach a certain level of percolation. At a low volume fraction of fibers, for example, when a certain level of percolation is needed for the mortar to be efficient as a selfsensing material [26], high fiber rupture would necessitate a higher volume fraction of fibers; since fiber rupture is unavoidable during mixing, a good mixing sequence would minimize the effect.

Fiber bundles in the fibrous mortar are caused either by initial clusters that have not been broken during the mixing sequence, or formation of clusters due to excessive mixing that would cause the fibers to coalesce, as stated previously [8]. The creation of clusters would change the morphology of the conductive volume. Also, based on percolation theory, this would reduce the efficiency of fibers in doping impedance [27].

A higher impedance would result from fiber rupture, poor fiber distribution or poor fiber dispersion. Any of these effects is indicative of poor efficiency of the mixing sequence in dispersing fibers. AC impedance measurement, then, is an efficient macro-scale tool to assess the mixing efficiency in dispersing fibers. In the following study, the efficiency of mixing sequences in dispersing fibers is examined from two different 
perspectives: the conventional statistical approach assessing the impedance, standard deviation and reproducibility values used in several previous studies, and a physical approach based mainly on the Nyquist plot of the material to determine the efficiency of the mixing process in dispersing fibers.

\subsection{Statistical approach}

In this approach, as mentioned previously, lower resistance, smaller standard deviation and a lower reproducibility factor $\mathrm{k}$ means a more efficient mixing sequence. At a low fiber volume fraction, standard deviation, and reproducibility factor for different mixing protocols at low mixing energy, as shown in Fig. 13, give a direct indication as to the reliability of the mixing sequences. The high factor k presented by Wet mix 2 reduces the credibility of its results. The One-step mixing sequence is the poorest-performing mixing protocol in terms of standard deviation values. Considering that the One-step method is the mixing sequence with the longest fiber mixing time, a previous study [8] mentioned the possible effect of coalescence of fibers, coupled with fiber rupture, that would decrease the efficiency of the One-step method in the case of fibrous mortar. Even though the Dry mix technique includes the same mixing time $(t=3$ minutes), it is possible that the prior dispersion with dry components could have reduced fiber clusters, leading to a difference in the impedance and standard deviation values of the two methods.

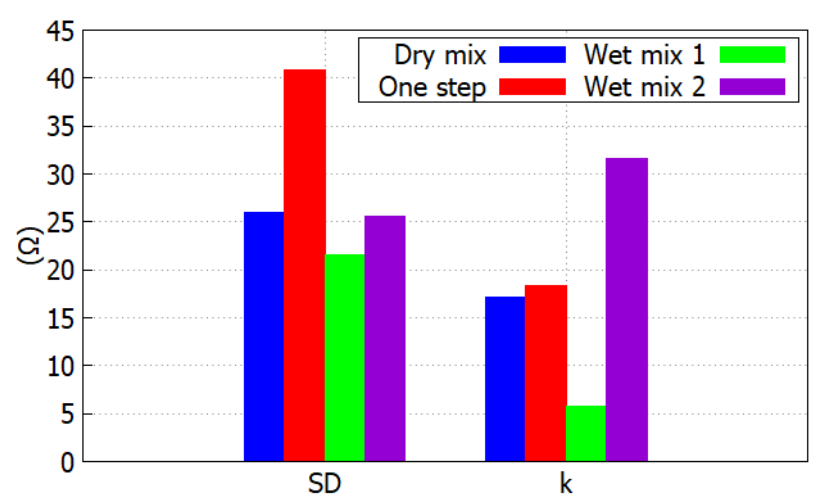

(a)

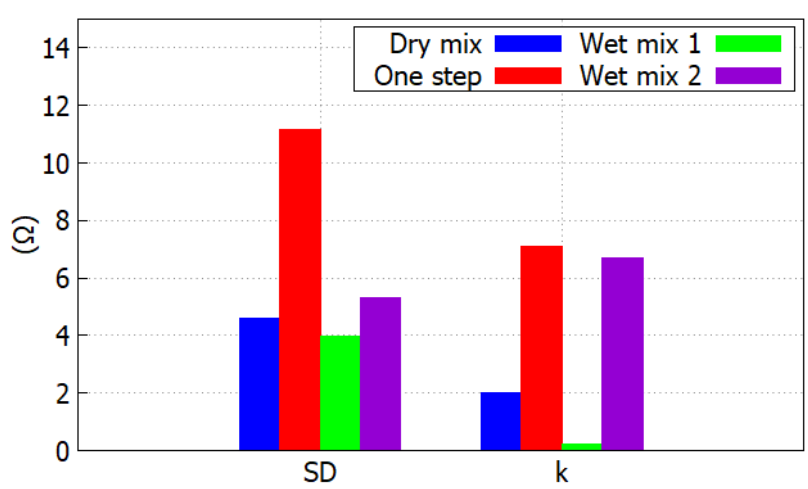

(b)

Fig. 13: Standard deviation and reproducibility factor of low-fiber mortar with different mixing sequences for (a) real impedance and (b) imaginary impedance

Compared to low mixing energy, resistance values with high mixing energy were significantly different. Extending the mixing time at low velocity increased the real and imaginary impedance for the different 
mixing sequences - i.e., the fibers are less efficient in reducing impedance by creating conductive paths, related to a poor distribution of fibers within the specimens. In addition, extending the mixing time at low velocity significantly increased the standard deviation values of measured impedance. This is the reflection of poor homogeneity between specimens, initially resulting from a reduced homogeneity in the total mixed volume. The same approach as used previously could be applied here to explain this impedance and standard deviation increase (excessive fiber rupture, and possible coalescence).

Increasing the mixing velocity in the final phase of mixing, instead of extending the mixing time, had varying results. Compared to the extended mixing time, impedance was slightly decreased, and standard deviation values were slightly reduced as well. Compared to the extended mixing time at low velocity, it showed lower impedance values, for different mixing sequences, and lower standard deviation values as well. The effect of alternating low and high mixing velocity is explained by Stroeven in a previous study [9]. Low velocity serves to distribute fiber agglomerations within the matrix, and high shear velocity serves to disperse agglomerated fibers already distributed in the matrix volume. This could explain the reduction of impedance compared to extended mixing time by ensuring better cluster dispersion. The cluster breakage effect is also coupled with fiber rupture, which would explain the higher impedance values compared to low mixing energy, due to reduced length of the conductive fillers.

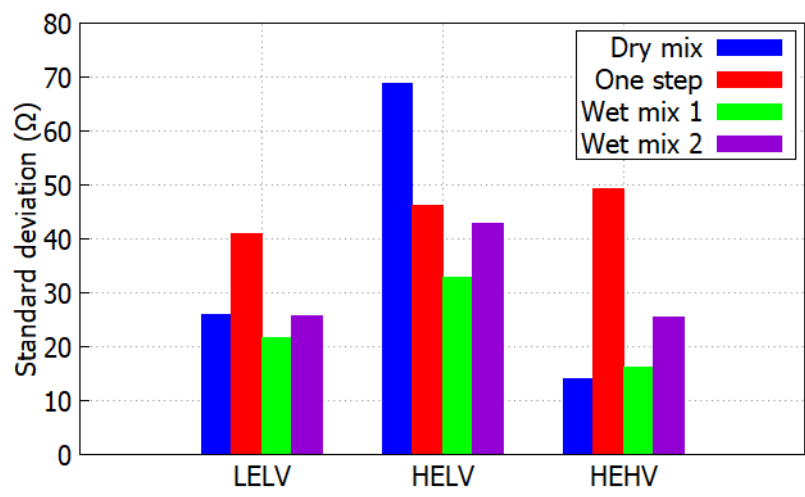

(a)

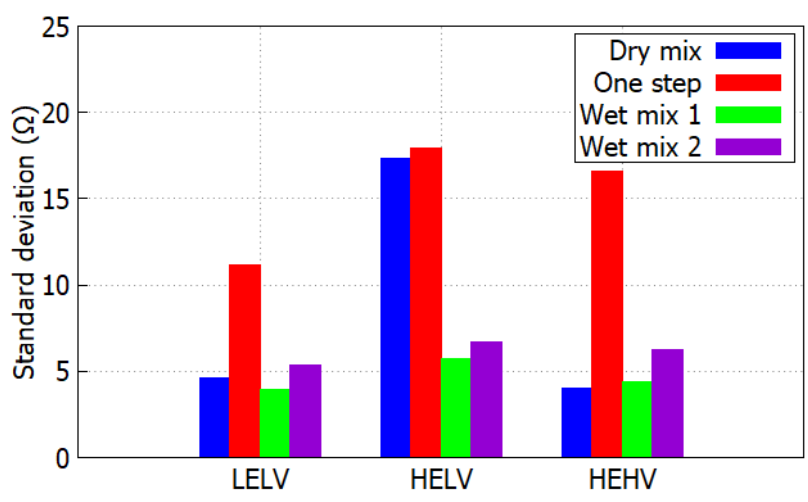

(b)

Fig. 14: Standard deviation of low-fiber mortar with different mixing energies for (a) real impedance and (b) imaginary impedance 
In the presence of a high carbon volume fraction, the real and imaginary impedance are significantly reduced by conductive filler percolation. Differences between mixing sequences were drastically reduced by a high carbon fiber volume fraction. In the presence of high carbon fiber dosage, the matrix impedance is considerably more immune to any possible effect of the mixing sequence. Impedance values remained low, and quasi-constant throughout the frequency range, and imaginary impedance presented moderate peak values at relaxation frequencies. Regarding the mixing energy effect in the case of high-fiber mortar, increasing the mixing energy increased the impedance, without any significant reduction in standard deviation. In order to understand the differential effects of mixing protocols for two fiber volume fractions, we must first understand the role played by the fibers: in the presence of $0.5 \%$ of carbon fibers, percolation of paths is established, and any potential fiber rupture or coalescence of fibers is not significant enough to cause a drastic increase in impedance, since it is overcome by the high fiber content.

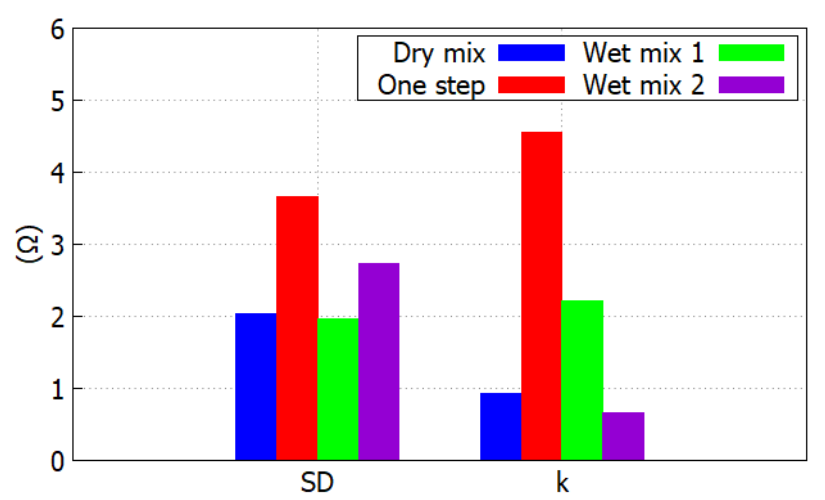

(a)

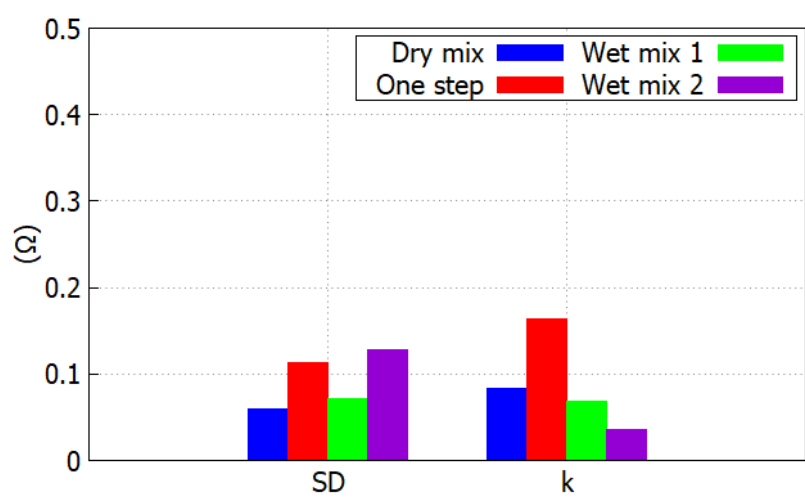

(b)

Fig. 15: Standard deviation and reproducibility factor of high-fiber mortar with different mixing sequences for (a) real impedance and (b) imaginary impedance

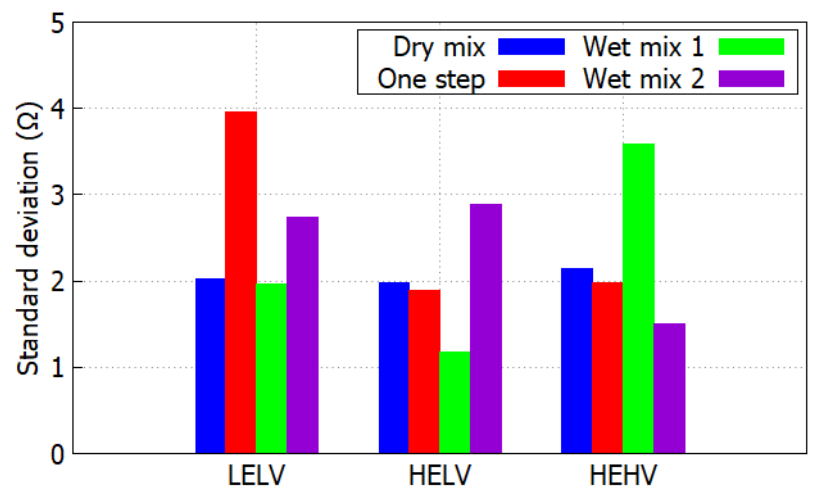

(a)

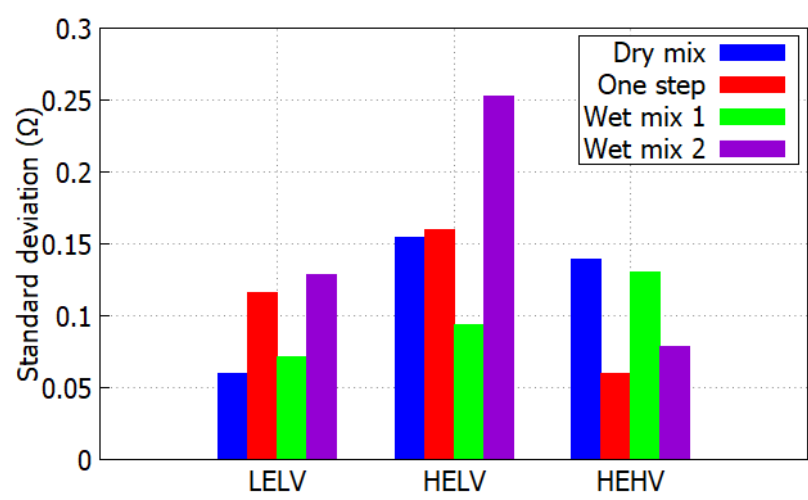

(b) 
Fig. 16: Standard deviation of high-fiber mortar with different mixing energies for (a) real impedance and (b) imaginary impedance

\subsection{Physical approach}

The physical approach, as explained previously, assesses the dispersion of carbon fibers by their efficiency in the transition from an insulating material to a conductive one. This approach is based on a simple relation between the Nyquist plot of the material and the creation of conductive paths. In a totally insulating mortar, the Nyquist plot would correspond to an R-C circuit with high resistance values, leading to a large semi-circle arc or diluted semi-circle arc. The presence of fibers, especially when percolated, would dope the impedance of the composite, creating parallel paths with lower resistance values, reducing the final equivalent circuit resistance values. The efficiency of carbon fibers as doping agents, as explained previously, is affected by fiber rupture, fiber dispersion and distribution: the better those fibers are dispersed, distributed through the matrix volume, and less broken by mixing protocols, the more the carbon fibers will reduce impedance. The progress from an insulating system to a percolated one is then assessed simply by studying the form of the Nyquist plot. In the following tables, for all mixing protocols, the diameter of the diluted semi-circle arc and the peak of its imaginary impedance are illustrated. At the same carbon fiber volume fraction, the mixing protocol and mixing energy had significant effects on the Nyquist plots of fibrous mortars. With a low carbon fiber volume fraction, changing the mixing protocol affected the efficiency of carbon fibers, especially with the One-step method that showed significant higher imaginary peaks at relaxation frequencies, and bigger Nyquist diagram diameter. This means that this mixing sequence was less efficient in ensuring a good distribution and dispersion for carbon fibers to lead to a conductive matrix. The same physical explanation used in section 4.1 related to fiber coalescence and breakage is applicable here. When we increased the mixing energy, the diameter value and imaginary peak values increased - i.e., the fibers were less effective in creating conductive paths to produce a transition to a more conductive system. This reduction in performance, explained in section 4.1, does not favor the increase in mixing energy to ensure better efficiency for carbon fibers. With high fiber content, the effect of the mixing sequence was significantly reduced, where impedance values are more homogenized between 
all mixing sequences. The effect of increasing mixing energy persisted on the Nyquist plots, in a moderate way compared to low carbon fiber content. Moreover, increasing mixing energy in both ways did not have the same effects: extending mixing time at low velocity increased diameter and peak diameters, while increasing the final mixing velocity produced a lesser such increase. This was related to possible better fiber dispersion, as explained in section 4.1 .

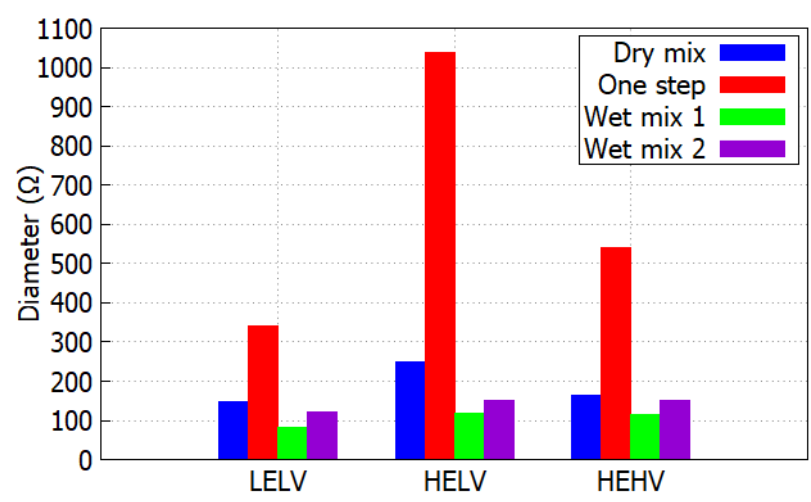

(a)

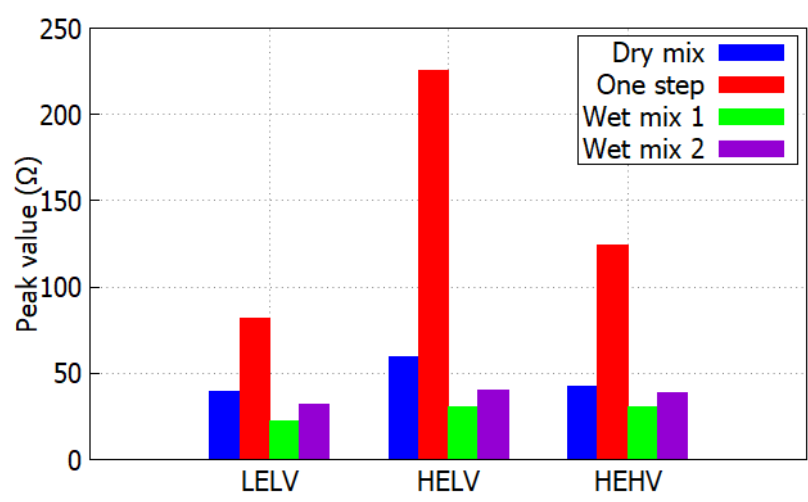

(b)

Fig. 17: Nyquist plot characteristics of low-fiber mortar with different mixing sequences (a) diameter value and (b) peak value

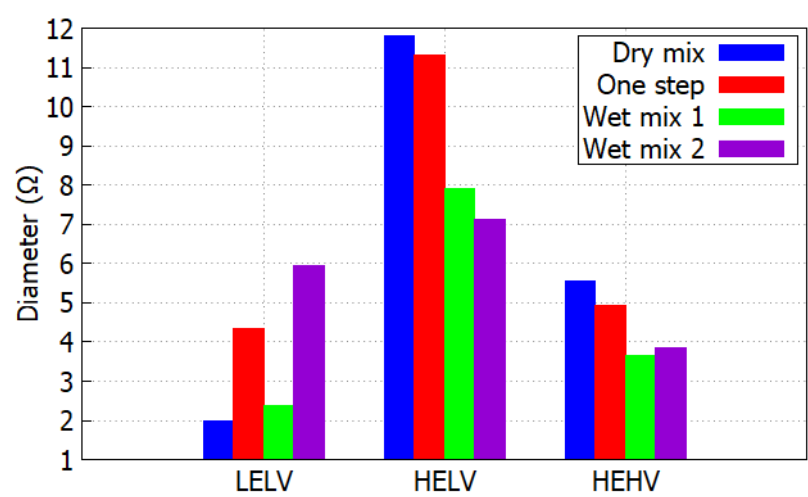

(a)

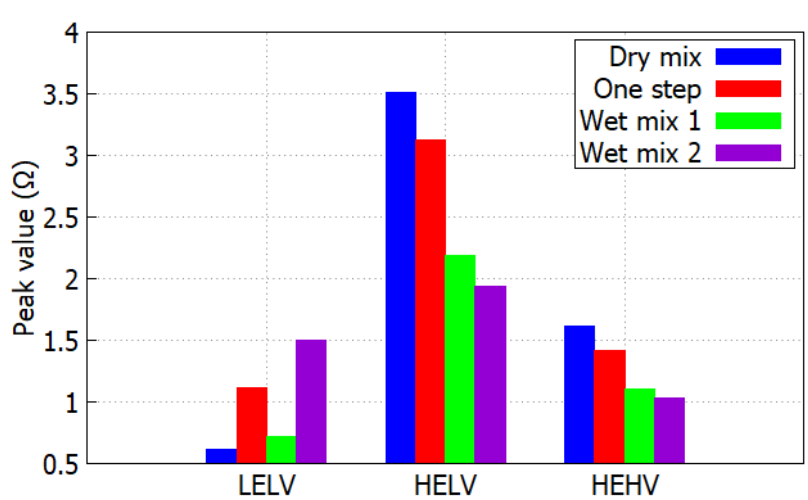

(b)

Fig. 18: Nyquist plot characteristics of high-fiber mortar with different mixing sequences (a) diameter value and (b) peak value

Both approaches converged on the same results: in mixing protocols, the One-step method at low mixing energy proved to perform poorly in dispersing fibers, where the corresponding mortar showed high impedance and high standard deviation. Increasing the mixing energy did not add positive effects on the dispersion of fibers, showing higher impedance, higher standard deviation, and bigger Nyquist plots at the 
same fiber volume fraction. Hence, to summarize the effect of mixing sequence, it appears to be a balance between fiber dispersion, fiber distribution and fiber rupture. In this study, low mixing energies with relatively short mixing time proved the most effective in promoting fiber efficiency in creating conductive paths.

\section{Conclusion}

An effective mixing sequence for fibrous mortar is a mixing sequence that guarantees equilibrium between fiber rupture, fiber dispersion and fiber distribution. This study addressed the efficiency of different mixing sequences in dispersing carbon fibers in a cementitious matrix, using AC impedance measurement with a statistical and a physical approach. The results can be summarized as follows:

- AC impedance measurement is an efficient tool to assess fiber dispersion in mortar. It can give a general indication about the homogeneity of fibrous mortar, by determining its impedance, which is directly related to the creation of conductive paths.

- The protocol of component addition is proved to influence the final impedance, and the homogeneity of the mortar: Dry mix protocol with 3 minutes of slow mixing at a velocity of $140 \mathrm{rpm}$ proved most reliable.

- Mixing energy was also a crucial factor, where mixing velocity and time had significant effects on the final impedance response: when the mixing energy is doubled, the impedance values are increased regardless of the order in which ingredients are added into the mix, proving to negatively affect fibers' efficiency in creating conductive paths.

- Mixing protocol effect depended on the percolation state of the carbon fibers: by increasing the fiber volume fraction from 0.1 to $0.5 \%$, the percolation status moved from around the theoretical percolation threshold to a saturated regime, and consequently the impedance of fibrous mortar become more stable i.e. the impedance showed smaller standard deviations and smaller gaps between different mixing protocols.

- Further work is needed concerning the quantification of fibers rupture, using 3D scanning for example, considering the importance of this information in the understanding of the mixing effect on the fibers 


\section{Credit authorship contribution statement}

Hamza Allam: Methodology, Formal analysis, Investigation, Writing - Original Draft.

François Duplan: Conceptualization, Methodology, Validation, Investigation, Supervision, Writing review.

Sofiane Amziane: Conceptualization, Methodology, Validation, Investigation, Supervision, Writing review \& editing,

Yves Burtshell: Validation, Supervision - review.

\section{Declaration of Competing Interest}

The authors declare that they have no known competing financial interests or personal relationships that could have appeared to influence the work reported in this paper.

\section{Bibliography}

[1] J. Cao and D.D.L Chung. Carbon fiber reinforced cement mortar improved by using acrylic dispersion as an admixture. Cement and Concrete Research, 31(11):1633-1637, November 2001.

[2] R. Siddique and A. Mehta. Effect of carbon nanotubes on properties of cement mortars. Construction and Building Materials, 50:116-129, January 2014.

[3] C. Wang, K.Z. Li, H.J. Li, G.S. Jiao, Jinhua Lu, and Dang-She Hou. Effect of carbon fiber dispersion on the mechanical properties of carbon fiber-reinforced cement-based composites. Materials Science and Engineering: A, 487(1):52-57, July 2008.

[4] B. Han, Y. Wang, S. Dong, L. Zhang, S. Ding, X. Yu, and J. Ou. Smart concretes and structures: A review. Journal of Intelligent Material Systems and Structures, 26(11):1303-1345, July 2015. Publisher: SAGE Publications Ltd STM.

[5] J. Donnini, T. Bellezze, and V. Corinaldesi. Mechanical, electrical, and self-sensing properties of cementitious mortars containing short carbon fibers. Journal of Building Engineering, 20:8-14, November 2018.

[6] H. Allam, F. Duplan, J. P. Clerc, S. Amziane, and Y. Burtschell. About electrical resistivity variation during drying and improvement of the sensing behavior of carbon fiber-reinforced smart concrete. Construction and Building Materials, 264:120699, December 2020.

[7] Y. Yang. Methods study on dispersion of fibers in CFRC. Cement and Concrete Research, 32(5):747-750, May 2002. 
[8] C. Wang, B. Li, Lei Peng, W. He, L. Zhao, and K. Li. CVD Treatment of Carbon Fibers and Evaluation of Their Dispersion in CFRC. Materials Sciences and Applications, 08(08):649, August 2017.

[9] P. Stroeven. Stereology of concrete reinforced with short steel fibres. HERON, 31 (2), 1986, Publisher: Delft University of Technology.

[10] F. D. E. Latief, I. H. Mohammad, and A. D. Rarasati. Digital 3D Microstructure Analysis of Concrete using X-Ray Micro Computed Tomography Sky Scan 1173: A Preliminary Study. IOP Conference Series: Materials Science and Engineering, 267(1):012020, 2017.

[11] Z. Wang, J. Gao, T. Ai, W. Jiang, and P. Zhao. Quantitative evaluation of carbon fiber dispersion in cement-based composites. Construction and Building Materials, 68:26-30, October 2014.

[12] V. C. Li and K. H. Obla. Effect of fiber length variation on tensile properties of carbon-fiber cement composites. Composites Engineering, 4(9):947-964, January 1994.

[13] A. Al-Dahawi, O. Oztürk, F. Emami, G. Yıldırım, and M. S,ahmaran. Effect of mixing methods on the electrical properties of cementitious composites incorporating different carbon-based materials. Construction and Building Materials, 104:160-168, February 2016.

[14] P. Xie, P. Gu, J.J. Beaudoin Electrical percolation phenomena in cement composites containing conductive fibers.

J. Mater. Sci., 31 (1996), pp. 4093-4097, 10.1007/BF00352673

[15] J. A. Orban, P. A. Parcevaux, and D. J. Guillot. Specific Mixing Energy: A Key Factor for Cement Slurry Quality. Society of Petroleum Engineers, January 1986.

[16] L. Y. Woo, S. Wansom, N. Ozyurt, B. Mu, S. P. Shah, and T. O. Mason. Characterizing fiber dispersion in cement composites using AC-Impedance Spectroscopy. Cement and Concrete Composites, 27(6):627-636, July 2005.

[17] P. Xie, P. Gu, Z. Xu, and J. J. Beaudoin. A rationalized A.C. impedance model for microstructural characterization of hydrating cement systems. Cement and Concrete Research, 23(2):359-367, March 1993.

[18] P. Xie, P. Gu, and J. J. Beaudoin. Electrical percolation phenomena in cement composites containing conductive fibers. Journal of Materials Science, 31(15):4093-4097, August 1996

[19] D. Chung. Dispersion of Short Fibers in Cement | Journal of Materials in Civil Engineering| Vol 17, No 4, 2005.

[20] J. M Torrents, T. O Mason, and E. J Garboczi. Impedance spectra of fiber-reinforced cement-based composites: a modeling approach. Cement and Concrete Research, 30(4):585-592, April 2000. 
[21] S. Wen and D. D. L Chung. Electric polarization in carbon fiber-reinforced cement. Cement and Concrete Research, 31(1):141-147, January 2001.

[22] A. Celzard, E. McRae, C. Deleuze, M. Dufort, G. Furdin, and J. F. Marêché. Critical concentration in percolating systems containing a high-aspect-ratio filler. Physical Review B, 53(10):6209-6214, March 1996. Publisher: American Physical Society.

[23] A. Belli, A. Mobili, T. Bellezze, and F. Tittarelli. Commercial and recycled carbon/steel fibers for fiber-reinforced cement mortars with high electrical conductivity. Cement and Concrete Composites, 109:103569, May 2020.

[24] D. D. L. Chung. Carbon fiber reinforced concrete. April 1992. Number: SHRP-ID/UFR-92-605.

[25] F. J. Baeza, O. Galao, E. Zornoza, and P. Garcés. Effect of aspect ratio on strain sensing capacity of carbon fiber reinforced cement composites. Materials \& Design, 51:1085-1094, October 2013.

[26] B. Han, X. Yu, and J. Ou. Butterworth-Heinemann, July 2014.Self-Sensing Concrete in Smart Structures.

[27] L. Berhan and A. M. Sastry. Modeling percolation in high-aspect-ratio fiber systems. I. Soft-core versus hard-core models. Physical Review E, 75(4):041120, April 2007. Publisher: American Physical Society. 